\title{
Novel Objective Time Derivative Obtained from Applying Riemannian Manifold of Riemannian Metrics to Kinematics of Continua
}

\author{
Z. Fiala \\ Institute of Theoretical and Applied Mechanics AS CR \\ Prosecka 76, 19000 Prague 9, Czech Republic \\ fiala@itam.cas.cz
}

\section{SUMMARY}

Large deformations are exposed from the viewpoint of differential geometry. This makes it possible to formulate kinematics of continua in a compact, coordinate-free way with clear geometrical meaning. In addition, more advanced concepts of Riemannian geometry bring interesting, entirely novel ideas to the very basic theory of large deformations. The primary aim of this paper is to present a brief outline of this approach and attract attention to these new ideas, quite unknown until now. At the end, a novel objective time derivative will be proposed.

\section{INTRODUCTION}

In order to consistently describe successive process of deformation, differential geometry is employed, which offers significantly more refined tools for description of finite deformations than matrix calculus. It was Noll who initiated a deep interest in the mathematical foundations of mechanics of continua, and, at present, Riemannian geometry has been employed in many papers concerned with theoretical aspects of the deformation of continua (Marsden and Hughes /11/, Giessen and Kollmann /4/, Stumpf and Hoppe /14/, Kadianakis $/ 9 /$ et al.).

In addition to presenting this standard view, the paper briefly outlines more advanced aspects of the kinematics of finite deformations, as they have appeared in the literature, although without attracting much notice. It is the book of Rougee /12/ in particular, which offers novel key ideas for a proper understanding of finite deformations, and closely related mathematical papers on geometry of infinite dimensional Riemannian spaces. By combining the Rougee's approach with this general mathematics, a new objective time derivative will be derived at the end. 


\section{PRELIMINARY INFORMATION - CONTINUUM MECHANICS AND RIEMANNIAN MANIFOLD}

Classically, the kinematics of continua is described by tensor fields on 3D Euclidean spaces $\mathbf{E}^{\mathbf{3}}$ using general curvilinear coordinate systems, but the more modern approach, considering Euclidean space as Riemannian manifold, predominates. For our purposes it suffices to characterize the Riemannian manifold as a set of points, with no privileged coordinate system endowed with metric, which enters the Riemannian manifold via the tangent space; for more, see Frankel /2/ and Schutz /13/. Making use of the geometrical entities (such as pull-back, push-forward, Lie derivatives, covariant derivative etc.) enables us to identify the actual geometrical content of the kinematics of finite deformations. The following three paragraphs offer a brief outline of Riemannian geometry in continuum mechanics. For more details, see Fiala /I/.

\subsection{Basic notions}

- A configuration of a simple body $B$, is a mapping $\ddot{\Phi}: I \times B \rightarrow \mathbf{E}^{3}$ parameterized by time from the interval $I=[0, T]$. The configurations at time 0 or at actual time $t$, called referential $R=\tilde{\Phi}(0, B)$ or spatial $S=\bar{\Phi}(t, B)$ configurations respectively, form Riemannian manifolds. The mapping $\bar{\Phi}$ then induces a mapping $\Phi: I \times R \rightarrow S$. We denote by $X$ points from $R$, and by $x$ points from $S$.

- The tangent space $T_{X} R$ is a linearized, infinitesimal neighbourhood of a point $X \in R$. It is a linear, finite-dimensional real vector space of all "infinitesimal material line elements" represented by vectors tangent at the point $X$ to curves lying in $R$.

- The cotangent space $T_{X}^{*} R$. It is again a linear, finite-dimensional real vector space of all "infinitesimal material surfaces" represented by covectors, which are quantities intimately related to gradients to functions at the point $X$. The covectors $a$ act as linear mappings $\langle a, u\rangle_{T_{y} R}$ of vectors $u$ to real numbers $\mathbf{R}$, and so the cotangent space is the dual space to the tangent space. Unlike the classical approach, making use of the dual space enables us to define the tensors on manifolds more clearly, and distinguish between vectors and covectors, contravariant and covariant tensors, being considered here as different objects. As above and below, the same applies to the spatial configuration.

- (p-q)-tensors ( $p$-contravariant, $q$-covariant) on a linear vector space $V$, with $V^{*}$ being its dual, are elements of the sets $T_{q}^{p}=T^{p} \otimes T_{q}=V \otimes \ldots \otimes V \otimes V^{*} \otimes \ldots \otimes V^{*}$ and $T_{0}^{0}=\mathbf{R}$. Here $V$ stands for some tangent space, $T_{X} R$ or $T_{x} S$, and $V^{*}$ for its corresponding cotangent space, $T_{X}^{*} R$ or $T_{x}^{*} S$.

- The key notion of Riemannian geometry is the metric, a positive-definite symmetric 2-covariant tensor $G$ defining the scalar product of two vectors $u, v \in T_{X} R: u \cdot v=G(u, v)$.

- The metric $G$ defines a mapping $\mathbf{G}: T_{X} R \rightarrow T_{X}^{*} R$ via the relation $\langle\mathbf{G} u, v\rangle_{T_{Y} R}=G(u, v)$. It assigns an associated covector $u^{\prime}$ to a vector $u$ : $u^{b}=\mathbf{G} u$ (and conversely an associated vector $a^{\prime}$ to a covector $a$ : 
$\left.a^{\prime}=\mathrm{G}^{-1} a\right)$. The so-called associated tensors $t^{\prime}, t^{b}$ to a (1-1)-tensor $t$ are (2-0)- and (0-2)-tensors respectively, defined by extending the mapping $\mathbf{G}$ to tensors. These operations correspond to raising and lowering indexes of components of tensors in classical approach. The metric $G$ also induces the scalar product on covector space, defined by means of the associated 2-contravariant tensor $G^{\mathbf{l}}$ : $a \cdot b \equiv G^{\sharp}(a, b)=G\left(a^{\sharp}, b^{\sharp}\right)$.

- A mapping $\Phi: I \times R \rightarrow S$ induces the tangent mapping (or deformation gradient $\mathbf{F}$ in other words) $T \Phi(=\mathbf{F}): T_{X} R \rightarrow T_{X} S$.

The tangent mapping defines push-forward $\Phi$. and pull-back $\Phi^{*}$ operations between corresponding spaces of tensors. These then, in a simple way, couple the description of deformation and stress state in the referential and spatial configurations: In fact, the description of the motion in the REFERENTIAL (SPATIAL) picture is obtained by pull-back (push-forward) of the spatial picture.

\subsection{Dual stress and strain tensors, dual time derivatives}

The various stress and strain tensors, and their objective time derivatives can be related to each other (Hill /7, 8/, Haupt and Tsakmakis /6/) via the stress power density:

$$
\pi_{t} \equiv \sigma_{t} \cdot d_{t}=\left\langle\sigma_{t}^{\sharp}, d_{t}^{b}\right\rangle_{T S^{*}}=\left\langle\sigma_{t}^{b}, d_{t}^{\sharp}\right\rangle_{T S},
$$

where $\sigma_{1}$ is the Cauchy stress (1-1)-tensor and $d_{1}$ is the rate-of-deformation (1-1)-tensor.

The Hill's result is obtained by pulling-back the spatial picture to the referential configuration, so that the referential stress power density reads

$$
\pi_{t}^{r e f}=\left\{\begin{array}{l}
\left\langle P_{t}^{\prime}, \partial E_{\prime}^{b}\right\rangle_{T R^{*}} \\
\left\langle K_{t}^{\prime}, \partial H_{l}^{\prime}\right\rangle_{T R}
\end{array}\right\}=\left\{\begin{array}{l}
P_{l} \cdot \partial E_{t} \\
K_{t} \cdot \partial H_{t}
\end{array}, \text { where } \partial\right. \text { stands for the material time derivative }
$$

In the above, the following two relations, playing the key role in the next paragraph, were employed

$$
\Phi^{*} d^{\prime}=\partial E^{b}\left(=\frac{1}{2} \partial C^{b}\right) \quad \Phi^{*} d^{\prime}=-\partial H^{\prime}\left(=-\frac{1}{2} \partial B^{\prime}\right)
$$

By pushing-forward the Hill's result back to spatial configuration Haupt and Tsakmakis obtained: 


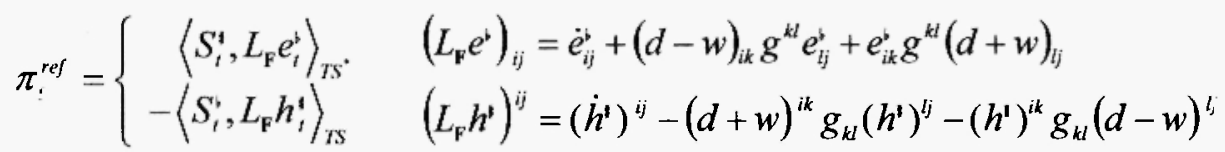

$L_{\mathrm{F}}=\Phi . \circ \partial \circ \Phi^{*}$ is the so-called Lie derivative (again $\mathbf{F} \equiv T \Phi$ ), and $w$ is vorticity. This dual time derivative (Oldroyd derivative), obtained from material derivative exactly the same way as the corresponding dual stress and strain tensors, is naturally objective.

$S=J \sigma$ is the WEIGHTED CAUCHY stress (1-1)-tensor (or KIRCHHOFF), and Jacobian $J$ (being scalar) is the determinant of the tangent mapping transformation $J=\operatorname{det}(\partial \Phi / \partial X) \sqrt{\operatorname{det}(g) / \operatorname{det}(G)}$.

$G$ (resp. $g$ ) denotes the metric on $R$ (resp. $S$ ) (i.e. the scalar product on $T_{X} R$ (resp. $T_{x} S$ )).

Tangent space: $1^{\text {st }}$ group

REFERENCE configuration: pulling back $g$ and $S^{4}$

$$
\begin{aligned}
& C^{\natural}=\bar{\varphi}^{*} g \\
& E=\frac{1}{2}(C-I) \\
& P^{\prime}=\Phi^{*} S^{\natural}
\end{aligned}
$$$$
\text { the associated RIGHT CAUCHY-GREEN deformation (0-2)-tensor }
$$$$
\text { the GREEN-ST.VENANT strain (1-1)-tensor (Lagrangian strain tensor) }
$$
the associated SECOND PIOLA-KIRCHHOFF stress (2-0)-tensor

\section{SPACE configuration: pushing forward $G$}

$$
c^{b}=\Phi, G
$$

$e=\frac{1}{2}(i-c)$ the ALMANSI-HAMEL strain (1-1)-tensor (Eulerian strain tensor)

- The following relations hold: $E^{\natural}=\Phi^{*} e^{\natural}, e^{\natural}=\Phi . E^{\natural}$.

\section{Dual space: $2^{\text {nd }}$ group}

REFERENCE configuration: pulling back $g^{\prime}$ and $-S^{b}$

$$
\begin{aligned}
& B^{s}=\Phi^{*} g^{\prime} \\
& \left.H=\frac{i}{2}(i)-I\right) \\
& K^{b}=-\Phi^{*} S^{b}
\end{aligned}
$$$$
\text { the PIOLA strain (1-1)-tensor }
$$

\section{SPACE configuration: pushing forward $G^{\prime}$}

$$
\begin{aligned}
& b^{\prime}=\Phi \cdot G^{\prime} \\
& h=\frac{1}{2}(i-b) \\
& -S^{\bullet}
\end{aligned}
$$$$
\text { the associated LEFT CAUCHY-GREEN deformation (2-0)-tensor }
$$$$
\text { the FINGER strain (1-1)-tensor }
$$
the (covariant) NEGATIVE WEIGHTED CAUCHY stress (0-2)-tensor

- Also, the following relations hold: $H^{*}=\Phi^{*} h^{\prime}, h^{4}=\Phi, H^{*}$. 
Table

Triplets of related strain, stress tensors and time derivatives

\begin{tabular}{|c|c|c|c|}
\hline Configuration & STRAIN TFNSORS & STRESS TENSORS & TIME DFRIVATIVES \\
\hline $\mathbf{1}^{\text {st }}$ groun & (covariant) & (contravariant) & \\
reference & GRFEN-ST.VENANT & $2^{\text {NI }}$ PIOLA-KIRCHHOFF & MATERIAL \\
space & AI.MANSI-HAMEL & KIRCHHOFF & OLDROYD \\
\hline $2^{\text {nd }}$ groun & (contravariant) & (covariant) & MATERIAL \\
reference & PIOLA & NEGATIVE CONVECTED & OLDROYD \\
\hline space & FINGER & NEGATIVE KIRCHHOFF & \\
\hline
\end{tabular}

\section{ADVANCED INFORMATION - RIEMANNIAN MANIFOLD OF RIFMANNIAN METRICS}

In summary, here are the main points of the previous paragraphs required later on:

- Finite deformations of the continua at the referential point $X$ are described by any of two deformation tensors $C^{b}(3)$ or $B^{\prime}(5)$.

- Their time derivatives $\partial C^{b}$ or $\partial B^{4}$, in progress of deformation, are obtained by pulling-back the corresponding associated tensors of the rate-of-deformation tensor $d^{\circ}$ or $d^{\text {: }}$ (see (2)).

At this point, some comments are still in order on why the deformation tensors, instead of strain tensors are more fitting for the description of the process of finite deformations. The answer reflects the very nature of the difference between finite and small deformations: Provided we split the deformation $x=\Phi(X)=X+u(X)$, for two successive deformations $X \rightarrow x_{1} \rightarrow x_{2}$, the following holds: $x_{2}=\Phi_{2} \circ \Phi_{1}(X)=\Phi_{2}\left(x_{1}\right)=\Phi_{2}\left(X+u_{1}(X)\right)=X+u_{2}\left(X+u_{1}(X)\right)+u_{1}(X)$. In case of small deformations one neglects all the terms of the second order in magnitude, and so the relation takes the form $x_{2} \approx X+u_{2}(X)+u_{1}(X)$, i.e. the diffeomorphism $\Phi$ acts as identity mapping $x=\Phi(X) \approx X$, and the conception of diffeomorphisms changes into that of fields. Similarly, for the deformation gradient $T \Phi=I+T u \approx I$, and for transformations of vectors and covectors: $v=T \Phi(V) \approx V, A=T \Phi^{\circ}(a) \approx a$. The conception of small deformations thus identifies tangent and cotangent spaces in reference, and spatial configurations. In particular, the metric tensors are equal $g \approx G$, and the objective time derivative is replaced by simple material time derivative $L_{\mathbf{F}}=\Phi_{*} \circ \partial \circ \Phi^{*} \approx \partial$. Infinitesimal variation $u(X)$ around identity mapping $\Phi_{0}(X)=x \approx X$ at the point $x=\Phi_{0}(X)$ (i.e. linearization of mapping $\Phi$ in other words) results in substituting fields for diffeomorphisms, and enters the theory of small deformations via 
infinitesimal variation of the metric $g=G$. It is the strain tensors $e \sim E, h \approx H$ that represent this infinitesimal variation of metric. Now, $c^{b} \approx C^{b}$ and $b^{*} \approx B^{\prime}$, and the relations (2) read: $\partial C^{b}=2 \partial E^{b} \approx 2 d^{b}$ and $\partial B^{\#}=2 \partial H^{\#} \approx-2 d^{\#}$.

On the other the hand, in the case of finite deformations the deformation process no longer keeps moving inside the tangent linear space $T_{c}, \mathbf{M}$ (see later on), as in the case of small deformations, and the finite difference between initial and terminal deformation tensors provides the same piece of information about deformation, as Euclidean distance between starting and ending points about the whole trajectory of a particle does: that means no information! Consequently, the deformation process at each material point $X$ should be described not by time dependent strains, but by a trajectory in the manifold $\mathbf{M}=\operatorname{Met}(R)$ of all possible deformation tensors (relative to reference configuration).

A fundamental observation of Rougee /12/ made it possible for him to significantly broaden the analysis of the process of finite deformations. He realized that the quantities $\partial C^{b}$ in fact constitute tangent vectors to the manifold $\mathbf{M}$ at the particular point $C^{b}$, chosen at the actual moment of time $t$. With the assistance of the relation $\partial C^{b}=2 \Phi^{*} d^{b}$ he introduced a scalar product on the tangent space $T_{i} \mathbf{M}$, so that the manifold $\mathbf{M}$ became Riemannian manifold. He managed to do this by extending the usual scalar product of vectors, defined on $T_{x} S$ by the metric $g$, to a scalar product of 2-tensors (see also (1)). In particular, for the rate-ofdeformation tensor $d^{b}$ he obtained: $\left.a^{n \cdot} \cdot a^{n}\right|_{g, x} ^{n}=g^{i k} g^{j l} d_{k j}^{1} d_{l i}^{2}$. As the diffeomorphism $\Phi$, is actually an isometry (a metric preserving diffeomorphism), he introduced the scalar product on the tangent space $T_{1} \mathbf{M}$ via the relation: $\left.\partial C^{\prime \prime} \cdot \partial C^{\prime 2}\right|_{c, i}=\Phi_{i}^{*}\left(d^{\prime \prime} \cdot d^{\prime 2}\right)$, where $\partial C^{b^{i}} \in T_{r}, \mathbf{M}, C^{b}=\Phi^{*}(g), B^{\prime}=\left(C^{b}\right)^{-1}$ and $\partial C^{b \prime}=2 \Phi_{,}^{*}\left(d^{b i}\right)$. Carrying out the pull-back operation, Rougee eventually obtained the metric on $\mathbf{M}$ (i.e. the scalar product on the tangent space $T_{\mathrm{t}} \mathbf{M}$ ):

$$
\left.\partial C^{\prime \prime 1} \cdot \partial C^{\prime 2}\right|_{1 \cdot x}=\frac{1}{4} B^{i k} B^{j l} \partial C_{k j}^{1} \partial C_{l i}^{2}
$$

Do not be confused by considering the deformation tensors $C^{b}$ as points of the Riemannian manifold $\mathbf{M}$, and their material time derivatives $\partial C^{b \prime} \in T_{1} . . M$ as vectors lying in the corresponding tangent space $T_{4} . . M$, at a particular point $C^{b}$ of $\mathbf{M}$ ! As we shall see in the next paragraphs, such a viewpoint offers far-reaching implications for the description of kinematics of finite deformations.

First, one can define time derivative of a tensor fields over $S$, via the covariant derivative of vector fields over $\mathbf{M}$. For a vector $V \in T, \mathbf{M}$ and a vector field $U$ over $\mathbf{M}$, the covariant derivative reads:

$$
\left(\nabla_{V} U\right)_{i j}=\left(\frac{\delta U}{\delta V}\right)_{i j}-\frac{1}{2}\left(V_{i l} B^{i k} U_{k j}+U_{i l} B^{u} V_{k j}\right), \text { where }\left(\frac{\delta U}{\delta V}\right)_{i j} \equiv \frac{d}{d q} U_{i j}\left(C^{k}+q V\right) l_{q=0} .
$$

Let $C: I \rightarrow \mathbf{M}$ denote a smooth curve, then derivative of vector field $U$ along the curve reads 


$$
\left(\frac{D}{D t} U\right)_{i j}=\left(\nabla_{\alpha^{\cdot}} U\right)_{i j}=\partial U_{i j}-\frac{1}{2}\left(\partial C_{i l} B^{k k} U_{k j}+U_{i l} B^{u} \partial C_{k j}\right) \text {, since } \frac{\delta U}{\delta\left(\partial C^{*}\right)}=\partial U \text {. }
$$

As now $\partial C^{b}=2 \Phi_{t}^{*} d^{\cdot}$, pushing the above derivative forward to the spatial configuration, one obtains

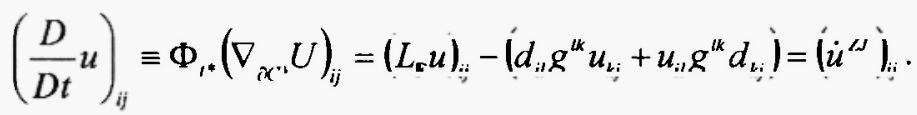

$u=\Phi_{,^{*}} U$ is any spatial 2-covariant symmetric tensor field over $S$ corresponding to $U$ over $\mathbf{M}$. The resulting time derivative is the Zaremba-Jaumann derivative (Rougee /12/). If we interpret parameter $t$ as time and the curve $C_{,}^{b}$ as a deformation process taking place at point $X$, the underlying mathematical structure of the Riemannian manifold $\mathbf{M}$, based on the metric (7), then unambiguously selects the only one objective time derivative (8). Note also that $\dot{g}^{\not J}=0$, or equivalently $D C^{b} / D t=0$.

Second, the geometrical structure of the manifold $\mathbf{M}$ enables us to clarifythe geometrical meaning of logarithmic strains by relating them to geodesics (Rougee /12/, APPENDIX).

Third, the manifold $\mathbf{M}$ can be split (Freed and Groisser /3/) into volumetric and shape submanifolds: $\mathbf{M} \equiv \operatorname{Vol}(R) \times \operatorname{Met}_{\mu}(R)$. Whereas the space $\operatorname{Vol}(R)$ is flat, the space $\operatorname{Met}_{\mu}(R)$ has nonzero curvature (negative), resulting in the dependence of deformation processes on the trajectory $C_{i}^{k}$ in $\mathbf{M}$. In particular, here seem to lie the problems with the existing use of logarithmic strains in modelling of constitutive relations.

\section{DISCUSSION AND NOVEL PROPOSAL OF TIME DERIVATIVE}

Provided we eliminate the restriction of deformation processes to a single material point $X \in R$, which is the case of Rougee /12/, we have slightly to modify the above theory. Now, the Riemannian metric is a tensor field $C^{k}$ of deformation tensors over the referential configuration $R$, and the corresponding manifold $\mathbf{M}$ of such Riemannian metrics is an infinite dimensional Riemannian manifold (Freed and Groisser /3/, GillMedrano and Michor /5/, Kriegel and Michor/10/). The metric (7) should then be modified by

$$
\left\langle U^{\prime}, U^{2}\right\rangle_{c^{\prime},}=\left.\int_{R} U^{1} \cdot U^{2}\right|_{C^{\cdot}, X} \mathrm{dVOL}{ }_{X}\left(C^{b}\right)=\left.\int_{R} \frac{1}{4} B^{i k} B^{j l} U_{k j}^{1} U_{l i}^{2} \sqrt{\operatorname{det}\left(C^{b}\right)}\right|_{X} \mathrm{~d} X
$$

Now, due to the additional multiplicative term $\sqrt{\operatorname{det}\left(C^{\prime}\right)}$ (which appears quite natural from the viewpoint of the relation (1) and that which immediately follows), the covariant derivative reads 


$$
\left(\nabla_{V} U\right)_{i j}=\left(\frac{\delta U}{\delta V}\right)_{l j}-\frac{1}{2}\left(V_{i l} B^{l k} U_{k j}+U_{l l} B^{l k} V_{k j}\right)+\frac{1}{4}\left(B^{k l} V_{l k} U_{i j}-B^{k l} V_{l o} B^{a p} U_{p k} C_{i j}+V_{l j} B^{k l} U_{l k}\right),
$$

and so for the objective time derivative of a spatial symmetric 2-covariant tensor field $u$ one obtains:

$$
\left(\frac{D}{D t} u\right)_{i j}=\left(\dot{u}^{\prime J}\right)_{i j}+\frac{1}{2}\left(g^{k l} d_{l k} u_{i j}-g^{k l} d_{l o} g^{o p} u_{p k} g_{i j}+d_{i j} g^{k l} u_{l k}\right)
$$

\section{CONCLUSION}

The approach sketched above, initiated by Rougee /12/, offers a great number of entirely novel ideas in the kinematics of finite deformations and deserves further scrutiny. To this end, the mathematical theory of infinite dimensional Riemannian manifolds of Riemannian metrics, as described in papers cited in the previous paragraph, will no doubt prove helpful. For a starter, novel objective time derivative (10) with clear geometrical origin has been proposed. As the time derivative should represent the rate of change of quantities attached to the points $X \in R$, the new objective time derivative seems to be promising. In fact, $D g / D t=3 / 2 \cdot d^{\natural}$ (cf. $L_{\mathrm{F}} g=2 d^{\natural}$ ) and $D C^{\natural} / D t=3 / 4 \cdot \partial C^{\natural}$, contrary to the Zaremba-Jaumann derivative, for which these derivatives are zeros.

\section{ACKNOWLEDGEMENT}

The research was conducted in the framework of the research plan AV0Z2071913. The support of the Grant Agency of the Czech Republic through the grant GA CR 103/03/0581, and of the Academy of Sciences of the Czech Republic through the project K1010104 is gratefully acknowledged. Especially, I would like to express my gratitude to Professor Rougee for valuable discussions on his book.

\section{REFERENCES}

1. Z. Fiala, Theory of finite deformations and differential geometry, in: Euromech Colloquium 430: Formulations and constitutive laws for very large strains, J. Plesek (Ed.), Prague (2002) 37-51.

2. T. Frankel, The geometry of physics. An introduction, Cambridge University Press (1997).

3. D.S. Freed and D. Groisser, The basic geometry of the manifold of Riemannian metrics and its quotient by the diffeomorphism group, The Michigan Mathematical Journal 36, 323-344 (1989). http://proiecteuclid.org/Dienst/Repository/1.0/Disseminate/euclid.mmj/1029004004/body/pdf. 
4. E. Giessen and F.G. Kollmann, On the mathematical aspects of dual variables in continuum mechanics. Part 1: Mathematical principles, ZAMM, 76(8), 447-462 (1996). Part 2: Applications in nonlinear solid mechanics, ZAMM, 76(9) 497-504, (1996).

5. O. Gill-Medrano and P.W. Michor, The Riemannian manifold of all Riemannian metrics, The Quarterly Journal of Mathematics 42, 183-202 (1991). http://www.mat.univie.ac.at/ michor/rie-met.ps

6. P. Haupt and Ch. Tsakmakis, On the application of dual variables in continuum mechanics, Continuum Mechanics and Thermodynamics, 1, 165-196 (1989).

7. R. Hill, On constitutive inequalities for simple materials, Journal of the Mechanics and Physics of Solids, 16, 229-242 (1968).

8. R. Hill, Aspects of invariance in solid mechanics, in Advances in Applied Mechanics 18, C.-S. Yih (Ed.), Academic Press (1978) 1-75.

9. N. Kadianakis, On the geometry of Lagrangian and Eulerian descriptions in continuum mechanics. ZAMM 79(2), 131-138 (1999).

10. A. Kriegel and P.W. Michor, The convenient setting of global analysis, Mathematical Surveys and Monographs 53, The American Mathematical Society, chap. IX, par. 45, 487-497 (1997). http://www.ams.org/online_bks/surv53/

11. J.E. Marsden and T.J.R. Hughes, Mathematical foundations of elasticity, Dover Publications, New York (1983).

12. P. Rougee, Mecanique des grandes transformations, Mathematique \& Applications 25, Springer (1997).

13. B. Schutz, Geometrical methods of mathematical physics, Cambridge University Press (1999).

14. H. Stumpf and U. Hoppe, The application of tensor algebra on manifolds to nonlinear conti-nuum mechanics - Invited survey article, ZAMM 77(5), 327-339 (1997).

\section{APPENDIX: A GEOMETRICAL MEANING OF THE LOGARITHMIC STRAIN TENSOR}

The geometrical structure of the manifold $\mathbf{M}$ makes it possible to clarify geometrical meaning of the logarithmic strains by relating them to geodesics. In general, geodesic is a curve connecting two points of a manifold by the shortest possible way, or equivalently, it is a curve of constant velocity, i.e. zero acceleration. In our case of manifold $\mathbf{M}$ with the metric defined by (7) [not by (9)!], for curve $C^{\natural}: I \rightarrow \mathbf{M}$ to be a geodesic, it has to satisfy:

$$
\left(\nabla_{i} \cdot \partial C^{k}\right)_{i j} \equiv \partial^{2} C_{i j}-\partial C_{i l} B^{l k} \partial C_{k j}=C_{i l} \partial\left(B^{k k} \partial C_{k j}\right)=0
$$

and the equation of geodetics reads 


$$
\partial\left(B^{i k} \partial C_{k j}\right)=-\partial\left(\partial B^{i k} C_{k j}\right)=0
$$

Consequently, during the deformation process the referential tensor $D=\Phi_{t}{ }^{*}(d): D_{j}^{l}=\frac{1}{2} B^{l k} \partial C_{k j}$ to the spatial rate-of-deformation tensor $d$ is constant. Let $D=\sum_{i} \Lambda_{i} p_{l} \otimes p^{\prime}$ be its spectral decomposition, where $\Lambda_{i}$ are proper numbers, $p_{i}$ proper vectors. The deformation process $C_{t}^{b}$ following a geodesic curve can thus be expressed in general as

$$
t \rightarrow C_{t}^{b}=\sum_{i} \exp \left[2_{i}\left(t-t_{0}\right)+\lambda_{i}\right] p^{i} \otimes p^{i}
$$

The manifold $\mathbf{M}$ is thus being divided into submanifolds $\mathbf{M}_{\mathbf{B}}$, made up of those deformation tensors, for which linearly independent directions $\mathbf{B}=\left\{p_{l}\right\}$ are mutually orthogonal. On each submanifold the deformation process takes precisely the above form. On the other hand, for any two metrics states $C_{t_{1}}^{b}, C_{t_{2}}^{b}$ there always exist submanifolds $\mathbf{M}_{\mathbf{B}}$ containing them both, and so they can be joined by a geodesic curve (Rougee /12/)

$$
C_{t}^{i}=\sum_{i} \exp \left[2 L_{i}\left(t-t_{1}\right) /\left(t_{2}-t_{1}\right)\right] \Delta^{i} \otimes \Delta^{\prime}
$$

Now, $L_{i}$ and $\Delta_{i}$ are defined via the tensor $\hat{\hat{C}_{j}^{i}}=\left(B_{i_{1}}^{\prime}\right)^{i k}\left(C_{t_{2}}^{\prime}\right)_{k i}$, relating the deformed state at the moment $t_{2}$ to that of $t_{1}$. Let $\hat{C}=\sum_{i} l_{i}^{2} \Delta_{i}^{\prime} \otimes \Delta^{\prime}$ be its spectral decomposition, then the logarithmic strain tensor $\hat{L}=\frac{1}{2} \ln \hat{C}=\sum_{i} L_{i} \Delta_{i} \otimes \Delta^{i}$, where $L_{i}=\ln l_{1}$. Contrary to other strain measures, only the logarithmic strain is able to couple meaningfully two remote deformations states, since it compares them as being placed on a geodetic, i.e. via a well defined deformation process unambiguously defined by its outside points (metric states $C_{t_{1}}^{b}$ or $C_{t_{2}}^{b}$ ) and corresponding tangent vectors pointing from one point to the second one. 